\title{
Harmonic analysis approach to solar cycle prediction and the Waldmeier effect
}

\author{
K. Petrovay \\ Eötvös University, Department of Astronomy, \\ Pf. 32, H-1518, Budapest, Hungary \\ email: K.Petrovay@astro.elte.hu
}

\begin{abstract}
It is demonstrated that, in addition to the precursor method, harmonic analysis approach to solar cycle prediction is also strongly conditioned by the Waldmeier effect.
\end{abstract}

Keywords. Sun: activity

\section{Introduction}

The current unusually deep and extended solar minimum has rekindled interest in solar cycle prediction. The apparently most successful approach to prediction has been the precursor method, based on the correlation of certain solar parameters around or before solar minimum with the amplitude of the following maximum. However, in an important paper Cameron \& Schüssler (2007) recently argued that most of these methods may be ultimately a simple consequence of Waldmeier's well known effect consisting in a correlation between the rise rate of a cycle and its maximal amplitude. This casts doubt on the feasibility of true cycle-to-cycle prediction, carried out without relying on indicators of the incipient new cycle.

These problems with the precursor methods suggest that it might be worth taking a second look at an alternative, time-honoured approach to the problem of solar cycle prediction: harmonic analysis. The first attempt to decompose the sunspot number series into a superposition of periodic functions, and then use it to extrapolate the series into the future was due to Kimura (1913). His list of periods already include a large number of values in the range 7 to 15 years; the physical reality of these periods, however, was doubted already by contemporary researchers. The approach most commonly used in harmonic analysis today, the power spectrum, was first taken for the sunspot number series by Cole (1973). In the following we take a fresh look at the power spectrum of the sunspot number series including data for the last few cycles and consider the question to what extent the resulting periods can be considered real physical periods and whether they are stable enough to serve as a basis for solar cycle prediction. Our findings indicate that harmonic analysis results are not free either from the strong influence of the Waldmeier effect.

\section{Power spectra of the sunspot number record}

Figure 1 presents the power spectrum of the smoothed annual sunspot number series for the whole period covered by the "official" record, i.e. 1749-2008.

Conspicuous peaks can be noticed at the first harmonic (5.5 years) and subharmonic (22 years) of the dominant 11-year peak. This is of course expected as in any series of asymmetrical cycles of variable amplitude such harmonics will naturally appear. 


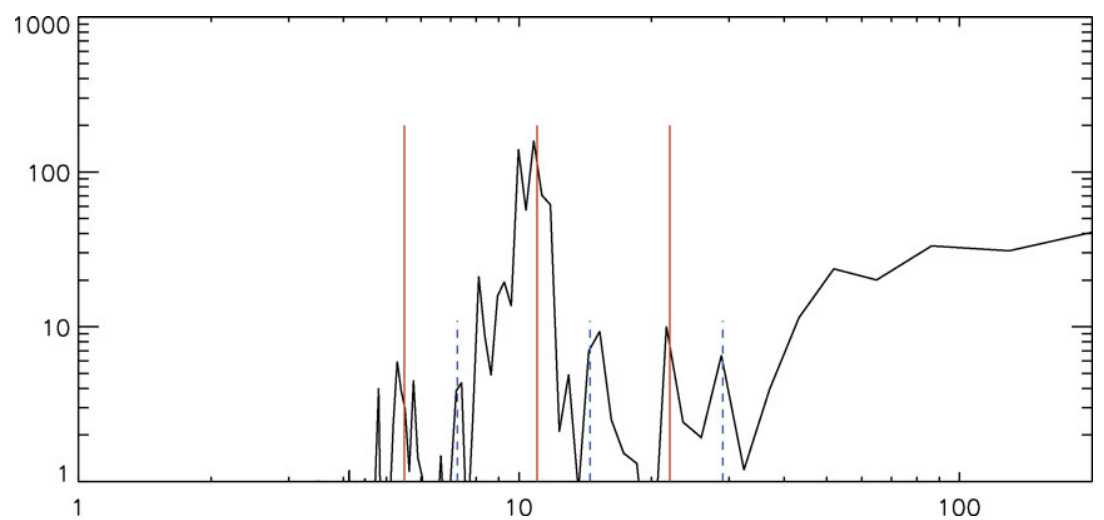

Figure 1. Power spectrum of the smoothed monthly sunspot number series for the period 1749-2008. Solid vertical bars mark the 11-year period, its first harmonic and subharmonic; dashed vertical bars are drawn at a fiducial period of 14.5 years, its harmonic and subharmonic.

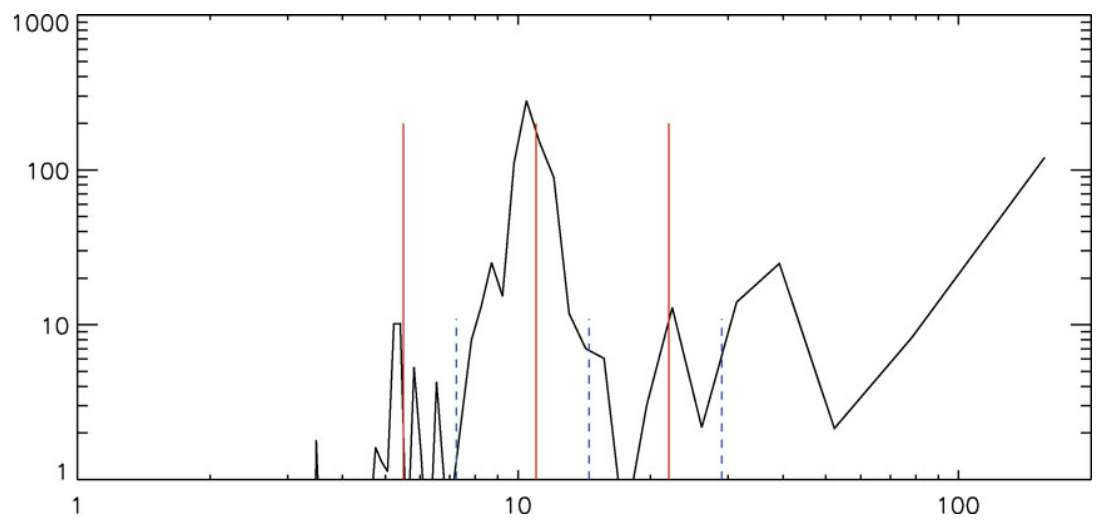

Figure 2. Same as Fig. 1 for the period 1850-2008.

What is more surprising is that, in addition to the finite natural width of the 11-year peak, with sidelobes, a separate secondary peak appears near a period of 14.5 years. The reality of this secondary period seems to be confirmed by the presence of further distinct peaks at the corresponding harmonic and subharmonic periods.

At first sight this finding might be taken as evidence for the physical reality of some decadal periods additional to the 11-year base period. However, constraining the time interval considered to data more recent than 1850, from which time the sunspot number series is generally considered to be more reliable, the 14.5-year secondary peak and its harmonics completely disappear (Fig. 2).

Does this mean that the apparent secondary period is due to unreliable early observations? Probably not: Figure 3 indicates that the appearance of the 14.5-year secondary peak in the complete series is almost entirely due to the strong predominance of this period (and its harmonic) in the interval 1783-1835, covering the Dalton minimum, consisting of three consecutive unusually weak cycles, when the "normal" 11-year mode of operation was completely suppressed. 


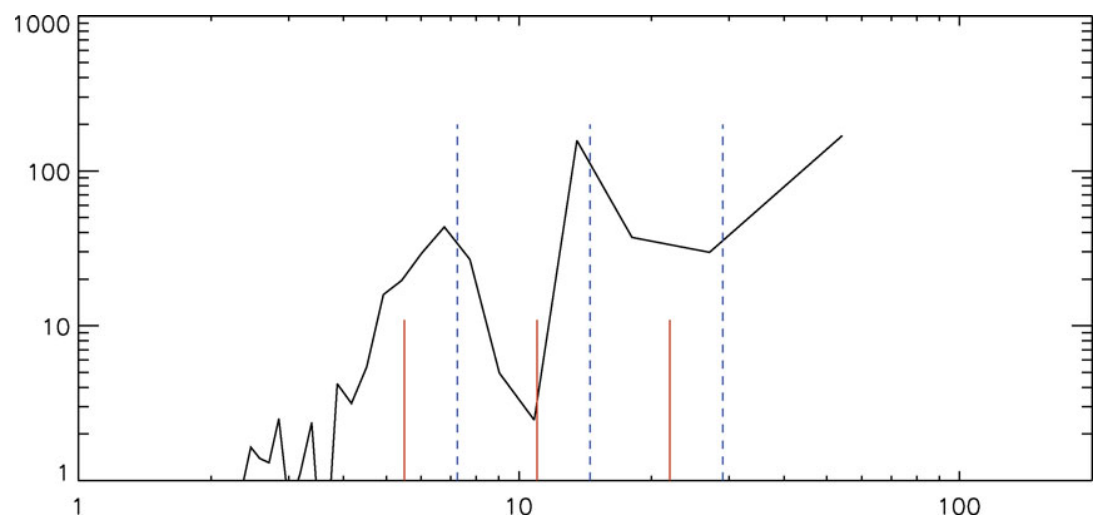

Figure 3. Same as Fig. 1 for the period 1783-1835.

\section{Interpretation}

Two possible interpretations arise regarding the findings of the previous section. On the one hand, the Sun may have been operating in a different mode during the Dalton minimum, the cycle length being 14.5 years instead of the usual 11 years. Indications that during the much deeper Maunder minimum the cycle period may have been as long as 22 years or so (Usoskin \& Mursula 2003) would seem to lend some plausibility to this assumption.

Figure 4, however, supports an alternative explanation. This plot shows the well known inverse correlation between cycle length and amplitude, which in turn is the consequence of the strong inverse correlation between rise time and cycle amplitude (Waldmeier effect), combined with a much weaker or nonexistent correlation between decay time and amplitude. It is clear that cycles 4 to 7 , covering the period in Fig. 3, all fall in the right hand side of the plot and are therefore characterized by a longer than average cycle length. The effect is even stronger in the case (not plotted here) when, instead of the usual minimum to minimum definition, cycle length is defined as the interval between successive maxima: indeed, the interval between the maxima of cycles 4 and 5 exceeded 17 years (Usoskin \& Mursula 2003, see Table 1 in Usoskin \& Mursula 2003).

The cycles around the Dalton minimum, then, seem to lie at the low amplitude (or long period) end of a continuum representing the well known cycle length-amplitude relation, ultimately explained by Waldmeier effect. There seems to be no evidence for a separate mode of operation of the dynamo during the Dalton minimum.

\section{Conclusion}

The predictive skill of harmonic analysis is well known to be limited but this is usually attributed to secular changes in the periods of modes and their amplitudes in the harmonic spectrum (e.g. Kane 2007). Recently there has been significant advance in the understanding of such secular changes for centennial cycles. For the Gleissberg cycle the results of Kolláth \& Oláh (2009) indicate a slow linear secular increase in the period. For the 210-year Suess cycle McCracken \& Beer (2008) present further evidence for the temporally intermittent nature of this marked peak in the spectrum of solar proxies. The Suess cycle seems to have a role in regulating the recurrence rate of grand 


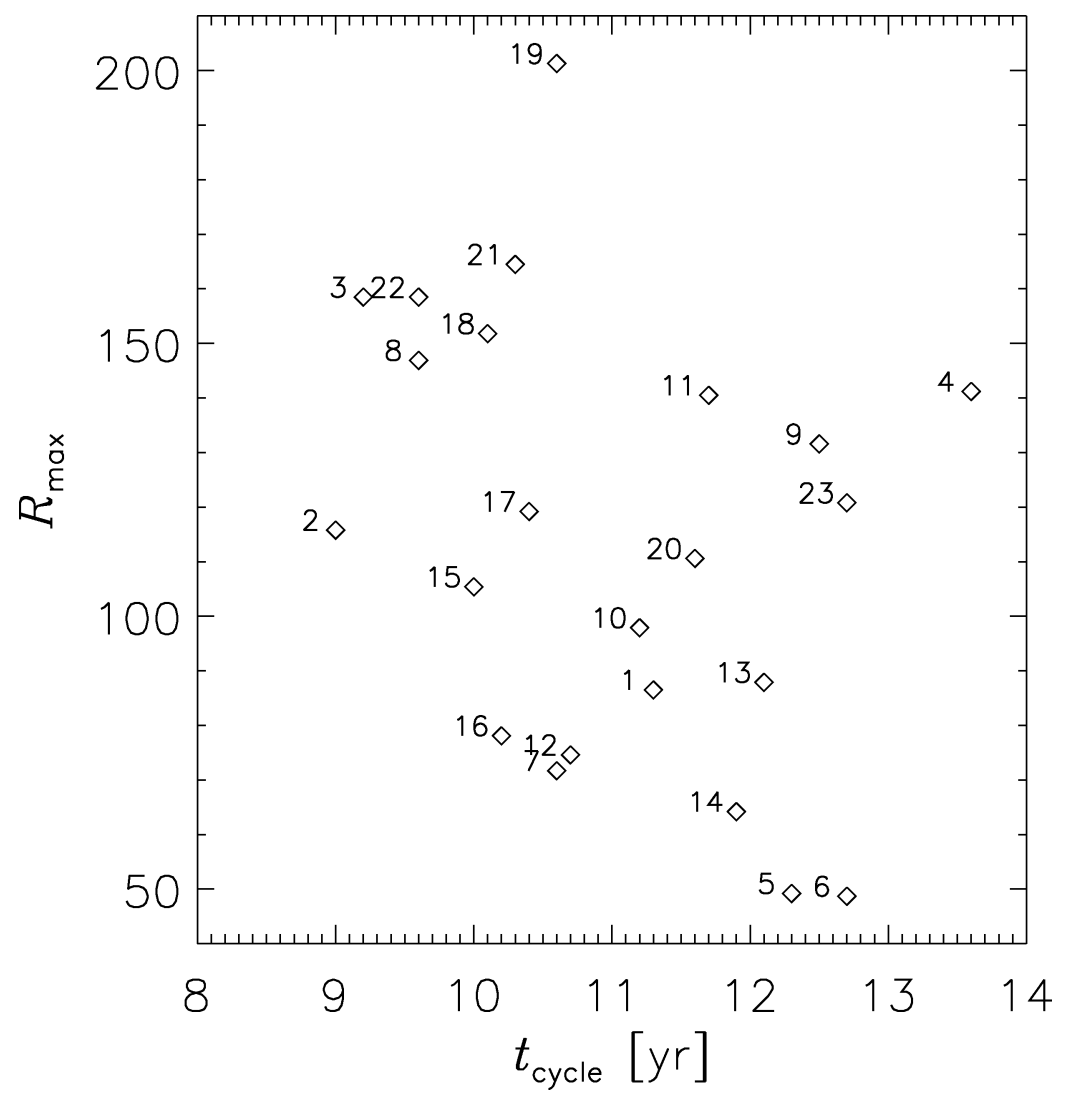

Figure 4. Plot of cycle length vs. cycle amplitude for solar cycles 1 to 23 .

minima. Grand minima, in turn, only seem to occur during $<1$ kiloyear intervals ("Spörer events") around the minimum of the $\sim 2400$-year Hallstatt cycle.

These results may raise the hope that with a better understanding of centennial and millennial variations of solar activity long term activity changes may ultimately be successfully predicted on the basis of the harmonic analysis approach. However, our findings in this paper regarding the role of the Waldmeier effect imply that even if centennial/millennial scale variations may be statistically predicted, there may still be fundamental obstacles in the way of a successful prediction of decadal scale cycle-to-cycle variations that are mankind's prime concern.

\section{Acknowledgement}

This research was supported by the Hungarian Science Research Fund (OTKA) under grant no. K67746 and by the European Commission through the RTN programme SOLAIRE (contract MRTN-CT-2006-035484).

\section{References}

Cameron, R. \& Schüssler, M. 2007, ApJ, 659, 801

Cole, T. W. 1973, Solar Phys., 30, 103 
Kane, R. P. 2007, Solar Phys., 246, 487

Kimura, H. 1913, MNRAS, 73, 543

Kolláth, Z. \& Oláh, K. 2009, A\&A, 501, 695

McCracken, K. G. \& Beer, J., 2008, in Proc. 30th International Cosmic Ray Conference, Vol. 1, UNAM, Mexico, p. 549

Usoskin, I. G. \& Mursula, K. 2003, Solar Phys., 218, 319 